Background: Current treatment approaches for autoimmune conditions comprise primarily of systemic immunosuppressants or cytokine blockade. The concentration of therapeutic molecules to the tissues that are the sites of autoimmune and inflammatory diseases is a promising approach with the potential to induce therapeutic benefit and avert risks associated with systemic immunotherapies. Pandion Therapeutics is developing a bifunctional antibody platform that can drive localized immune modulation by combining a "tether antibody" that targets a tissue of choice and "an effector end" that activates specific regulatory immune pathways to restore immune-homeostasis.

Objectives: Here we report the engineering of a skin-tethered PD-1 agonist and a skin-tethered CD39 that inhibit T cell activation and function and deplete local ATP, respectively, modulating different arms of the immune system in a tissue specific manner.

Methods: Biophysical assays were performed to characterize Skin-tethered immune effectors for drug-like properties and in vitro and in vivo assays for target binding, cellular activity and tissue specific-localization. Moreover, these bifunctionals were tested in pathway-relevant preclinical models such as Vitiligo and Contact Hypersensitivity.

Results: Biophysical characterization of the bifunctional molecules showed desired drug like properties including specificity, stability, and manufacturability. The skin tethered bifunctionals showed effector activity in in vitro assays and selectively localized to the skin. Skin localization strikingly correlated with a tether-dependent efficacy compared to a non-tether control.

Conclusion: We believe that this therapeutic approach has the potential to drive the resolution of cutaneous inflammation, providing an opportunity for developing new targeted therapies for autoimmune and inflammatory skin diseases.

Disclosure of Interests: Susmita Borthakur Shareholder of: Pandion Therapeutics Inc., Employee of: Pandion therapeutics Inc., Purvi Mande: None declared, Daniel Rios: None declared, Patrick Halvey: None declared, Angela Boisvert: None declared, Mlchael Rowe: None declared, Anisha Agrawal: None declared, Minasri Borah: None declared, Mike Cianci: None declared, Joanne L. Viney Shareholder of: Pandion therapeutics Inc., Consultant of: Harpoon, Finch, Quench, HotSpot, Employee of: Pandion Therapeutics Inc., Katalin KisToth: None declared, Kevin L. Otipoby Shareholder of: Pandion Therapeutics Inc., Employee of: Pandion Therapeutics Inc., Ivan Mascanfroni: None declared, Nathan Higginson-Scott Shareholder of: Pandion Therapeutics Inc., Consultant of: Mediar Tx, Employee of: Pandion Therapeutics Inc.

DOI: 10.1136/annrheumdis-2021-eular.1665

\section{AB0020 ACPA ILLUSTRATING THE IMPACT OF IGG FAB- GLYCOSYLATION ON TRANSPLACENTAL TRANSFER OF ANTIBODIES AND THEIR BINDING TO THE NEONATAL FC-RECEPTOR (FCRN)}

M. Volkov ${ }^{1}$, K. Van Schie ${ }^{1}$, A. Bondt ${ }^{2}$, T. Kissel ${ }^{1}$, M. Brinkhaus ${ }^{3}$, A. Bentlage ${ }^{3}$, C. Koeleman ${ }^{4}$, S. De Taeye ${ }^{3}$, R. Dolhain ${ }^{5}$, M. Wuhrer ${ }^{4}$, R. Toes ${ }^{1}$, G. Vidarsson ${ }^{3}$ D. Van der Woude ${ }^{1}{ }^{1}$ Leiden University Medical Center, Rheumatology, Leiden, Netherlands; ${ }^{2}$ Utrecht University, Biomolecular Mass Spectrometry and Proteomics, Utrecht, Netherlands; ${ }^{3}$ Sanquin Research, Immunoglobulin Research, Amsterdam, Netherlands; ${ }^{4}$ Leiden University Medical Center, Center for Proteomics and Metabolomics, Leiden, Netherlands; ${ }^{5}$ Erasmus Medical Center, Rheumatology, Rotterdam, Netherlands

Background: $F c$ neonatal receptor $(F c R n)$ is crucial for IgG half-life and transplacental transport. Different sites of IgG carry glycans which may affect binding to FcRn. While the effect of Fc-glycans has been investigated, the impact of Fab-glycosylation $(\sim 14 \% \lg G)$ on IgG-FcRn interaction remains unclear. Anti-citrullinated protein antibodies (ACPA) of rheumatoid arthritis patients exhibit remarkably high Fab-glycosylation ( $90 \%)$. This makes ACPA an ideal model to investigate how Fab-glycosylation influences IgG-FcRn interaction.

Objectives: To investigate the potential impact of IgG Fab-glycosylation on IgG transplacental transfer and interaction with FcRn.

Methods: To investigate transplacental transport of ACPA and total IgG, serum of ACPA-positive RA patients (mothers) as well as of healthy mothers and their respective newborns was analyzed. IgG Fab- and Fc-glycosylation was investigated with liquid chromatography and mass-spectrometry. Furthemore, ACPA monoclonal IgG were produced and glycoengineered to acquire several variants of the same monoclonal antibody differing only in their glycosylation profile. These glycovariants were then used to investigate the impact of Fab-glycans on the affinity of $\lg G$ for $\mathrm{FcRn}$. Surface plasmon resonance (SPR) and affinity chromatography were implemented.

Results: When measured in mothers' serum and cord blood samples, Fab-glycosylation of IgG antibodies was $20 \%$ lower in newborns compared to their mothers, which was observed for ACPA IgG, non-ACPA IgG in RA patients and total $\lg G$ of healthy controls (Figure 1). This may indicate that transplacental transfer of Fab-glycosylated antibodies is impaired. SPR results suggested that presence of Fab-glycans slightly lowered the affinity of IgG for FcRn. However, presence of Fab-glycans did not have a significant effect on the results of FcRn affinity chromatography. Together, these results suggest that Fab-glycans may impair association of IgG with FcRn, while dissociation likely stays intact.

Conclusion: Our results suggest that Fab-glycans inhibit IgG-FcRn binding which negatively affects the transplacental transfer of Fab-glycosylated IgG. The impact of Fab-glycosylation on IgG half-life requires further investigation.

Fab-glycosylation percentage of IgG in serum of mothers and their newborns
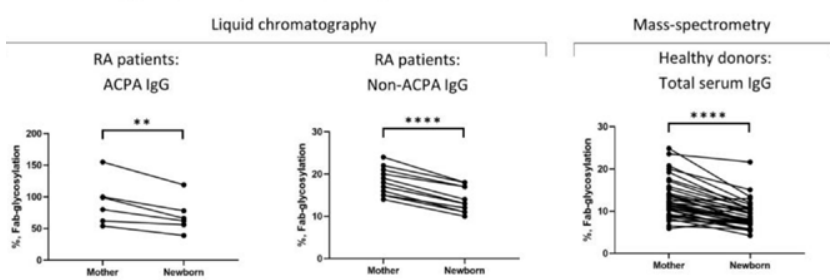

Figure 1.

Disclosure of Interests: Mikhail Volkov: None declared, Karin van Schie: None declared, Albert Bondt: None declared, Theresa Kissel: None declared, Maximilian Brinkhaus Grant/research support from: argenx, Arthur Bentlage: None declared, Carolien Koeleman: None declared, Steven de Taeye Grant/research support from: Genmab, Radboud Dolhain: None declared, Manfred Wuhrer: None declared, Rene Toes: None declared, Gestur Vidarsson: None declared Diane van der Woude: None declared DOI: 10.1136/annrheumdis-2021-eular.1727

\section{$\mathrm{AB} 0021$}

CHARACTERISATION OF AGE-ASSOCIATED B CELLS IN EARLY, DRUG NAÏVE RHEUMATOID ARTHRITIS

G. Vidal-Pedrola ${ }^{1}$, N. Naamane ${ }^{1}$, D. Scheel-Toellner ${ }^{2}$, A. Pratt ${ }^{1}$, A. Mellor ${ }^{1}$, J.

D. Isaacs ${ }^{1}$, A. E. Anderson ${ }^{1} .{ }^{1}$ Newcastle University, Translational and Clinical Research Institute, Newcastle upon Tyne, United Kingdom; ${ }^{2}$ University of Birmingham, Centre for Translational Inflammation Research, Birmingham, United Kingdom

Background: Rheumatoid arthritis (RA) is a chronic inflammatory disease of immune dysregulation affecting the joints. While $T$ cells play a recognised role in disease pathogenesis, the presence of autoantibodies years before the clinical onset of disease, and the efficacy of the B cell-depleting therapy rituximab, highlight a pathogenic role for these functionally diverse lymphocytes. A novel subset, termed age-associated $B$ cells (ABCs), are described as CD19 ${ }^{\text {high }}$ CD21 $\mathrm{CD}_{11 \mathrm{C}^{+}}$, with a high proportion expressing the transcription factor T-bet; they are elevated in murine models of autoimmunity and produce autoantibodies characteristic of autoimmune disease. A detailed characterisation of ABCs in early, drug naïve RA has not yet been conducted.

Objectives: We aimed to characterise peripheral blood (PB) and synovial fluid (SF) ABCs in patients suffering from early drug naïve RA. As a secondary objective we sought to determine whether this population differs between RA patients and age-matched early psoriatic arthritis (PsA; disease controls) and healthy controls.

Methods: Newly presenting early RA and other inflammatory arthritis patients, naïve to immunomodulatory treatment, were recruited from the Newcastle Early Arthritis Clinic. Patients with established RA ( $\geq 1$ year duration on treat ment and age-matched healthy controls were recruited in parallel. B cell sub-

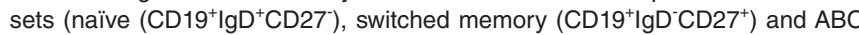
$\left.\left(\mathrm{CD} 19^{+} \mathrm{CD} 21^{-} \mathrm{CD} 11 \mathrm{c}^{+}\right)\right)$in $\mathrm{PB}$ and SF were characterised by flow cytometry. FACS sorted PB B cell subset gene expression profiles were assessed using a customised NanoString nCounter Human Immunology v2 Panel.

Results: Transcriptionally, $A B C$ differed from both naïve and switched memory $B$ cells. In keeping with published studies ABCs had an activated memory phenotype exemplified by elevated expression of CD69, CD80, and CD86, as well as Ki67, $\mathrm{HLA}$-DR, IgG and T-bet. Interestingly, ABC had high expression of the Fc Recepto Like (FCRL) family (FCRL1-5), and an inflammatory homing profile (high levels of CXCR3 and low levels of CXCR4 and CXCR5). We found no difference in the proportion of PB ABC between RA patients and control groups, and no association of $A B C$ frequencies with age. Focussing on RA $A B C$ s specifically, we observed elevated $\mathrm{ABC}$ frequencies in females, but no association with disease activity. In keeping with their chemokine receptor profile, cross-sectional analysis also demonstrated $A B C$ enrichment in SF compared to $\mathrm{PB}$, with SF frequencies higher in established than early disease. The transcriptome of $A B C$ s from early RA patients differed from both age-matched disease controls and healthy donors (Figure 1 next page). Conclusion: These data demonstrate that $A B C s$ have a unique, activated, class-switched proliferative phenotype that is transcriptionally distinct from switched memory and naïve B cells. Interestingly, at the transcriptome level early RA ABCs differ to their counterparts in health and other inflammatory arthritides, suggesting they may differ functionally and contribute to pathogenesis. Of note 